\title{
SINGULAR POINTS OF FUNCTIONS WHICH SATISFY THE PARTIAL DIFFERENTIAL EQUATION OF THE FLOW OF HEAT*
}

BY D. V. WIDDER

1. Introduction. It has frequently been pointed out that the function

$$
\begin{aligned}
U(x, y ; a, b) & =\frac{1}{(y-b)^{1 / 2}} e^{\mu}, & & (y>b), \\
& =0, & & (y \leqq b),
\end{aligned}
$$

where $\mu=-(x-a)^{2} /[4(y-b)]$, plays a rôle in the theory of the solutions of the equation

$$
\frac{\partial^{2} u}{\partial x^{2}}-\frac{\partial u}{\partial y}=0
$$

which is quite analogous to that played by the function

$$
\log \left[(x-a)^{2}+(y-b)^{2}\right]^{1 / 2}
$$

in the theory of harmonic functions. One would expect, therefore, to find a characterization of the function (1) similar to that given by Bôcher $\dagger$ for (3). It is the purpose of the present note to obtain such a characterization.

For brevity we designate a function as regular in a given region if it is continuous with its first derivatives there. The results to be proved are the following.

THEOREM 1. If $f(x, y)$ is a single-valued solution of (2) regular in the neighborhood of a point $(a, b)$ except at $(a, b)$, and is bounded, then $f(x, y)$ becomes regular at $(a, b)$ if its definition at this point is properly adjusted.

* Presented to the Society, December 27, 1929.

$\dagger$ M. Bôcher, Singular points of functions which satisfy partial differential equations of the elliptic type, this Bulletin, vol. 9 (1903), p. 455. See also O. D. Kellogg, On some thearems of Bôcher concerning isolated singular points of harmonic functions, this Bulletin, vol. 32 (1926), p. 664. Professor Kellogg has informed the author that Bôcher's principal theorem was also known to Schwarz. 
THEOREM 2. If $f(x, y)$ is a single-valued solution of (2) regular in the neighborhood of a point $(a, b)$ except at $(a, b)$, and if $f(x, y)$ is bounded on one side only, then $f(x, y)-L U(x, y ; a, b)$ is regular at $(a, b)$ provided the constant $L$ is properly chosen and the function is properly defined at $(a, b)$.

Our proof, like Bôcher's, employs a Green's function; but the method of proof must necessarily differ considerably from that of Bôcher since the level curves

$$
\frac{1}{(y-b)^{1 / 2}} e^{\mu}=\text { constant }, \quad \mu=-\frac{(x-a)^{2}}{4(y-b)},
$$

do not in this case consist of closed curves bounding regions with $(a, b)$ in the interior, but all pass through this point.

2. Green's Formula and Green's Function. In the present section we shall recall certain results from the classical theory of equation (2). Set

$$
F(u)=\frac{\partial^{2} u}{\partial x^{2}}-\frac{\partial u}{\partial y}, \quad G(u)=\frac{\partial^{2} u}{\partial x^{2}}+\frac{\partial u}{\partial y} .
$$

Then $G(u)$ is the adjoint of $F(u)$ and the equation

$$
G(u)=0
$$

is the adjoint of equation (2). Let $D$ be a regular* region with boundary $C$. If $u(x, y)$ and $v(x, y)$ are two functions continuous with their first and second derivatives in and on the boundary of $D$, then Green's formula is

$$
\int_{D} \int[v F(u)-u G(v)] d x d y=\int_{C} u v d x+\left(v \frac{\partial u}{\partial x}-u \frac{\partial v}{\partial x}\right) d y
$$

Here the contour integration is in the positive sense. $\dagger$ We now define the Green's function corresponding to a given rectangle.

Definition. Let the vertices $A, B, F, E$ of a rectangle $R$ have

* For present purposes the boundary may consist of a finite number of straight-line segments.

$\dagger$ Most of the results of this section will be found in E. Goursat, Cours d'Analyse, 1923, vol. 3, Chap. 29. In applying this formula to regular solutions of (2) and (4) we make use of the fact that such solutions are known to have continuous second derivatives. 
coördinates $\left(x_{0}, y_{0}\right),\left(x_{0}+p, y_{0}\right),\left(x_{0}+p, y_{0}+q\right),\left(x_{0}, y_{0}+q\right)$ respectively, $p$ and $q$ being arbitrary positive constants, and let $(\xi, \eta)$ be an arbitrary interior point. By the Green's function of this rectangle we mean a function $G(x, y ; \xi, \eta)$ which, considered as a function of $x$ and $y$ :

(a) satisfies (2) and is regular in the closed region $R$ except at $(\xi, \eta)$;

(b) has the form

$$
G(x, y ; \xi, \eta)=U(x, y ; \xi, \eta)+u(x, y)
$$

at $(\xi, \eta)$ where $u(x, y)$ satisfies (2) and is regular in the closed region $R$;

(c) is zero on the line segments $E A, A B, B F$.

The existence and uniqueness of this function follows from known results concerning a familiar boundary-value problem.* It may also be shown that $G$ satisfies the adjoint equation (4) considered as a function of $(\xi, \eta)$, and is regular except at the point $(x, y)$, where it has the form

$$
U(x, y ; \xi, \eta)+v(\xi, \eta),
$$

$v(\xi, \eta)$ being a solution of (4) regular throughout $R$. Moreover, $G$ vanishes on the sides $A E, E F, F B$ when considered as a function of $(\xi, \eta)$.

3. Three Lemmas.

Lemma 1. If $\phi(x, t)$ is a solution of (2) regular in $R$ except perhaps at an interior point $(a, b)$, then the integral

$$
\int_{C} \frac{\partial \phi}{\partial x} d y+\phi d x
$$

has a constant value $K$ when extended in the clock-wise sense over the contours $C$ of all rectangles lying in $R$ and containing $(a, b)$ as an interior point.

This follows from formula (5) by taking $u=\phi, v=1$, and $D$ the region between two of the rectangles considered. In particular, if $\phi$ is regular at $(a, b), K$ is zero.

Leмma 2. If $\phi(x, y)$ satisfies the conditions of Lemma 1 , is

* E. Goursat, loc. cit., p. 316. 
bounded at least on one side, and vanishes on the sides $E A, A B$, $B F$, then

$$
\lim _{h \rightarrow 0^{+}} \int_{a-k}^{a+k} \phi(x, b+h) f(x) d x=K f(a)
$$

for any function $f(x)$ continuous in $(a-k, a+k)$ and for any $k$ such that the points $(a-k, b),(a+k, b)$ lie in $R$.

Proof. Since $\phi(x, y)$ is bounded on one side it is possible to find a constant $H$ such that the function $\psi(x, y)=\phi(x, y)+H$ is a function of one sign in $R$. For definiteness we suppose it positive. As a result of Lemma 1 we have

$$
\begin{aligned}
\int_{a-k}^{a+k} \phi(x, b+h) d x & +\int_{b}^{b+h} \frac{\partial \phi}{\partial x}(a-k, y) d y \\
& +\int_{b+h}^{b} \frac{\partial \phi}{\partial x}(a+k, y) d y=K .
\end{aligned}
$$

We have here chosen the contour $C$ as the boundary of the rectangle whose vertices are $(a-k, b-h),(a+k, b-h),(a+k, b+h)$, $(a-k, b+h)$. It was unnecessary to include the complete contour since $\phi$ vanishes identically for $y<b$. This follows* since $\phi$ is zero on the sides $E A, A B, B F$, and is regular for $y<b$. If $h$ tends to zero we clearly have

or

$$
\lim _{h \rightarrow 0^{+}} \int_{a-k}^{a+k} \phi(x, b+h) d x=K,
$$

$$
\lim _{h \rightarrow 0^{+}} \int_{a-k}^{a+k} \psi(x, b+h) d x=K+2 k H .
$$

We shall first show that

$$
\lim _{h \rightarrow 0^{+}} \int_{a-k}^{a+k} \psi(x, b+h) f(x) d x=K f(a)+H \int_{a-k}^{a+k} f(x) d x .
$$

The desired result will follow from this equation by noting that

$$
\int_{a-k}^{a+k} \phi(x, b+h) f(x) d x=\int_{a-k}^{a+k} \psi(x, b+h) f(x) d x-H \int_{a-k}^{a+k} f(x) d x .
$$

\footnotetext{
* E. Goursat, loc. cit., p. 309.
} 
We employ the following identity:

(6)

$$
\begin{aligned}
I_{h}= & \int_{a-k}^{a+k} \psi(x, b+h) f(x) d x-K f(a)-H \int_{a-k}^{a+k} f(x) d x \\
= & \int_{a-\alpha}^{a+\alpha}[f(x)-f(a)] \psi(x, b+h) d x+f(a) \int_{a-\alpha}^{a+\alpha} \psi(x, b+h) d x \\
& -K f(a)+\int_{a+\alpha}^{a+k} \phi(x, b+h) f(x) d x \\
& +\int_{a-k}^{a-\alpha} \phi(x, b+h) f(x) d x-H \int_{a-\alpha}^{a+\alpha} f(x) d x .
\end{aligned}
$$

Here $\alpha$ is an arbitrary number for which $0<\alpha<k$. Given an arbitrary positive number $\epsilon$, we determine $\alpha$ so small that the following inequalities hold:*

$$
\begin{aligned}
\left|\int_{a-\alpha}^{a+\alpha} f(x) d x\right| & <\frac{\epsilon}{5 H}, \\
|f(x)-f(a)| & <\frac{\epsilon}{5(K+2 k H)},|x-a| \leqq \alpha, \\
\alpha & <\frac{\epsilon}{10 H|f(a)|} .
\end{aligned}
$$

With this choice of $\alpha$ equation (6) gives the inequality

$$
\begin{aligned}
\left|I_{h}\right| \leqq & \frac{\epsilon}{5(K+2 k H)} \int_{a-\alpha}^{a+\alpha} \psi(x, b+h) d x \\
& +|f(a)|\left|\int_{a-\alpha}^{a+\alpha} \psi(x, b+h) d x-K\right| \\
& +\left|\int_{a+\alpha}^{a+k} \phi(x, b+h) f(x) d x\right| \\
& +\left|\int_{a+k}^{a-\alpha} \phi(x, b+h) f(x) d x\right|+\frac{\epsilon}{5}
\end{aligned}
$$

We can now choose $h$ so small that the right-hand side of this

* $H$ may clearly be chosen positive and so great that $K+2 k H$ is positive. If $f(a)=0$, condition (c) becomes superfluous. 
inequality will be less than $\epsilon$. For we have shown that we can choose $h$ so small that the integral

$$
\int_{a-\alpha}^{a+\alpha} \psi(x, b+h) d x
$$

differs as little as we like from $K+2 \alpha H$, and hence is less than $K+2 k H$. For such values of $h$ the first term on the right-hand side of (7) is less than $\epsilon / 5$. The second term may be made to differ as little as we like from $2 \alpha H|f(a)|$ by choice of $h$, and hence made less than $\epsilon / 5$ by virtue of (c). The two remaining integrals of (7) approach zero as $h$ approaches zero since they are continuous functions of $h$ in the neighborhood of $h=0$ and since $\phi(x, b)=0$ in the intervals $a+\alpha \leqq x \leqq a+k, a-k \leqq x \leqq a-\alpha$. We may therefore determine $h$ so that each is in absolute value less than $\epsilon / 5$. It follows that $\left|I_{h}\right|<\epsilon$ for $h$ sufficiently small, and the lemma is established.

LEMмA 3. If $\phi(x, y)$ satisfies the conditions of Lemmas 1 and 2, and if $v(x, y)$ is a solution of (4) regular in $R$, then

$$
\begin{aligned}
\int_{C_{0}} \phi(x, y) v(x, y) d x+ & {\left[v(x, y) \frac{\partial \phi}{\partial x}(x, y)\right.} \\
& \left.-\phi(x, y) \frac{\partial v}{\partial x}(x, y)\right] d y=K v(a, b),
\end{aligned}
$$

where $C_{0}$ is the contour of any rectangle in $R$ with its sides parallel to the axes and including $(a, b)$ as an interior point, and where the integration is in the clockwise sense.

Proof. Consider the function $\phi(x, y+h)$ where $h$ is a small positive constant which, in the course of the proof, will be allowed to approach zero as its limit. This function is a solution of (2) regular in the rectangle whose vertices are $(a-k, b)$, $(a+k, b),(a+k, b+r),(a-k, b+r)$ provided $r, h$ and $k$ are chosen positive and sufficiently small; for, the point $(a, b-h)$ at which $\phi(x, y+h)$ may fail to be regular lies outside the rectangle. We may now apply Green's formula to this rectangle taking $u(x, y)=\phi(x, y+h)$ and $v(x, y)=v(x, y)$. Then

$$
\int_{a-k}^{a+k} \phi(x, b+h) v(x, b) d x
$$




$$
\begin{aligned}
& =\int_{b}^{b+r}\left[v(a-k, y) \frac{\partial \phi}{\partial x}(a-k, y+h)-\phi(a-k, y+h) \frac{\partial v}{\partial x}(a-k, y)\right] d y \\
& +\int_{a-k}^{a+k} \phi(x, b+r+h) v(x, b+r) d x \\
& +\int_{b+r}^{b}\left[v(a+k, y) \frac{\partial \phi}{\partial x}(a+k, y+h)-\phi(a+k, y+h) \frac{\partial v}{\partial x}(a+k, y)\right] d y .
\end{aligned}
$$

Now let $h$ approach zero. By Lemma 2 the first integral tends to $K v(a, b)$. The integrals on the right are continuous functions of $h$ in the neighborhood of $h=0$, so that their limits are obtained by setting $h=0$ in their integrands.

It is now an easy matter to complete the proof. By Green's formula the integral (8) extended over $C_{0}$ is equal to the same integral extended over the rectangle whose vertices are $(a-k$, $b-r),(a+k, b-r),(a+k, b+r),(a-k, b+r)$, where $r$ and $k$ are so chosen that the rectangle lies in $R$. But we have seen that $\phi(x, y) \equiv 0$ if $y<b$ so that the part of the contour for which $y<b$ may be neglected. The integral extended over the remainder of the contour, as we have just seen, is equal to $K v(a, b)$. The equation (8) is thus established.

4. Proof of the Theorems. Let $f(x, y)$ be a solution of (2) regular in the neighborhood of $(a, b)$ except at $(a, b)$. Choose the rectangle $R$ of Section 2 so that $f(x, y)$ is regular throughout $R$ except at $(a, b)$. Determine a solution $\bar{f}(x, y)$ of $(2)$ regular throughout $R$ and assuming the same values as $f(x, y)$ on the sides $E A, A B, B F$. If $f(x, y)$ is bounded at least on one side, then the function $\phi(x, y)=f(x, y)-\bar{f}(x, y)$ has the same property, and may be taken as the function $\phi$ of the lemmas.

Let $\left(x_{1}, y_{1}\right)$ be an arbitrary interior point of $R$ not the point $(a, b)$. Surround $\left(x_{1}, y_{1}\right)$ by a small rectangle with boundary $C_{1}$, with sides parallel to the axes, lying entirely in $R$, and such that $(a, b)$ is an exterior point. In a similar way construct a rectangle with boundary $C_{0}$ about $(a, b)$ and so small that all points of $C_{1}$ are exterior points. Let $C$ be the boundary of $R$. Form the Green's function $G(x, y ; \xi, \eta)$ for the region $R$. Fix the first pair of arguments at $\left(x_{1}, y_{1}\right)$, and let the second pair be variable. The functions $\phi(x, y)$ and $G\left(x_{1}, y_{1} ; x, y\right)$ are regular in the region $D$ bounded by the curves $C, C_{0}, C_{1}$, the former a solution of (2), the latter a solution of (4). If we apply Green's formula to these two functions in the region $D$ we obtain 


$$
\begin{gathered}
\int_{C_{0}} \phi(x, y) G\left(x_{1}, y_{1} ; x, y\right) d x+\left(G \frac{\partial \phi}{\partial x}-\phi \frac{\partial G}{\partial x}\right) d y \\
+\int_{C_{1}} \phi(x, y) G\left(x_{1}, y_{1} ; x, y\right) d x+\left(G \frac{\partial \phi}{\partial x}-\phi \frac{\partial G}{\partial x}\right) d y=0 .
\end{gathered}
$$

The integration is in the same sense over $C_{0}$ and $C_{1}$. The integral extended over $C$ disappears since either $\phi$ or $G$ vanishes on each side of the rectangle. By Lemma 3 , the first of these integrals has the value $\pm K G\left(x_{1}, y_{1} ; a, b\right)$. The second may easily be evaluated by known theory. For, we recall that, in the neighborhood of $\left(x_{1}, y_{1}\right), G\left(x_{1}, y_{1} ; x, y\right)=U\left(x_{1}, y_{1} ; x, y\right)+v(x, y)$, where $v(x, y)$ is a solution of (4) regular in the region bounded by $C_{1}$. Moreover.

and

$$
\int_{C_{1}} \phi v d x+\left(v \frac{\partial \phi}{\partial x}-\phi \frac{\partial v}{\partial x}\right) d y=0
$$

$$
\begin{gathered}
\int_{C_{1}} \phi(x, y) U\left(x_{1}, y_{1} ; x, y\right) d x+\left(-\phi \frac{\partial U}{\partial x}+U \frac{\partial \phi}{\partial x}\right) d y \\
= \pm 2 \pi^{1 / 2} \phi\left(x_{1}, y_{1}\right) .^{*}
\end{gathered}
$$

Hence $2 \pi^{1 / 2} \phi\left(x_{1}, y_{1}\right)= \pm K G\left(x_{1}, y_{1} ; a, b\right)$. As a function of $\left(x_{1}, y_{1}\right), G$ is a solution of (2), and we have

$$
\begin{aligned}
& \phi(x, y)= \pm \frac{K}{2 \pi^{1 / 2}} U(x, y ; a, b)+u(x, y) \\
& f(x, y)= \pm \frac{K}{2 \pi^{1 / 2}} U(x, y ; a, b)+u(x, y)+f(x, y) .
\end{aligned}
$$

We see that if $f(x, y)$ is bounded on both sides $K$ must be zero, for, otherwise the right-hand side of (9) would be unbounded, and we should have a contradiction. On the other hand, if $f(x, y)$ is unbounded on one side, $K$ can not be zero. If we choose the constant $L$ of Theorem 2 as $\pm K /\left(2 \pi^{1 / 2}\right)$, equation (9) serves to prove that theorem. Both theorems are thus completely established.

Bryn Mawr College

*E. Goursat, loc. cit., p. 311. 